\title{
A New Circuit Model for Spin-Torque Oscillator Including Perpendicular Torque of Magnetic Tunnel Junction
}

\author{
Hyein Lim, Sora Ahn, Miryeon Kim, Seungjun Lee, and Hyungsoon Shin \\ Department of Electronics Engineering, Ewha Womans University, 11-1 Daehyun-Dong, Seodaemoon-Gu, \\ Seoul 120-750, Republic of Korea
}

Correspondence should be addressed to Hyungsoon Shin; hsshin@ewha.ac.kr

Received 7 May 2013; Accepted 11 June 2013

Academic Editor: Mohindar S. Seehra

Copyright (C) 2013 Hyein Lim et al. This is an open access article distributed under the Creative Commons Attribution License, which permits unrestricted use, distribution, and reproduction in any medium, provided the original work is properly cited.

\begin{abstract}
Spin-torque oscillator (STO) is a promising new technology for the future RF oscillators, which is based on the spin-transfer torque (STT) effect in magnetic multilayered nanostructure. It is expected to provide a larger tunability, smaller size, lower power consumption, and higher level of integration than the semiconductor-based oscillators. In our previous work, a circuit-level model of the giant magnetoresistance (GMR) STO was proposed. In this paper, we present a physics-based circuit-level model of the magnetic tunnel junction (MTJ)-based STO. MTJ-STO model includes the effect of perpendicular torque that has been ignored in the GMR-STO model. The variations of three major characteristics, generation frequency, mean oscillation power, and generation linewidth of an MTJ-STO with respect to the amount of perpendicular torque, are investigated, and the results are applied to our model. The operation of the model was verified by HSPICE simulation, and the results show an excellent agreement with the experimental data. The results also prove that a full circuit-level simulation with MJT-STO devices can be made with our proposed model.
\end{abstract}

\section{Introduction}

Spin-torque oscillator (STO) is a promising new device which is based on the spin-transfer torque (STT) effect in magnetic multilayered nanostructure. According to the theory presented by Slonczewski [1] and Berger [2], spin-polarized current may produce precessing magnetization in the ferromagnetic material. Many experiments and researches have been carried out to support the theory about STO. Recently, interest in the magnetic tunnel junction (MTJ) which uses a thin dielectric spacer (e.g., $\mathrm{MgO}$ ) as is shown in Figure 1(a) is rapidly growing because it may overcome the limitation of small power in giant magnetoresistance (GMR) devices.

The effect of STT in MTJ-STO can be explained by Landau-Lifshitz-Gilbert (LLG) equation that represents the movement of the magnetization. LLG equation has been proved very useful in describing the precessional motion of magnetization in the ferromagnetic material. In this equation, spin torque consists of two components: in-plane torque $\left(a_{I}\right)$ and perpendicular torque $\left(b_{I}\right)$. In a metallic GMR device, the perpendicular torque is very small compared with the in-plane torque such that it can be safely ignored.
In MTJ devices, however, the perpendicular torque has a magnitude of about $30 \%$ of the in-plane torque. Thus, when an MTJ device is modeled, the perpendicular torque has to be considered.

Researches on STOs have been mostly focused on physical characteristics of the device with respect to the composing material and the structure whereas study on the application of STO devices to CMOS circuits is still very immature. One of the major obstacles to the integration of STO devices with CMOS circuits is the lack of circuit-level model of STO devices. Therefore, the model that represents accurate physical characteristics of STO has to be developed to accelerate the convergence of STO devices with CMOS technology.

In our previous work, physics-based GMR-STO circuit model was proposed $[3,4]$. In this paper, a new circuit model for MTJ-STO is proposed including the effect of perpendicular torque. The model is fully compatible with circuitlevel simulators such as SPICE. A current mirror circuit that contains an STO element and a multistage wideband amplifier was designed to verify the correctness and usability of our model in real-world circuit design. 


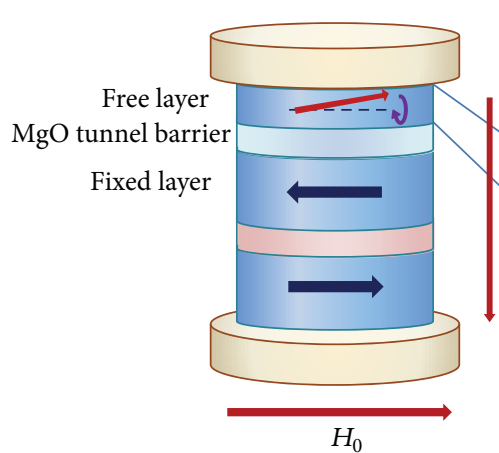

(a)

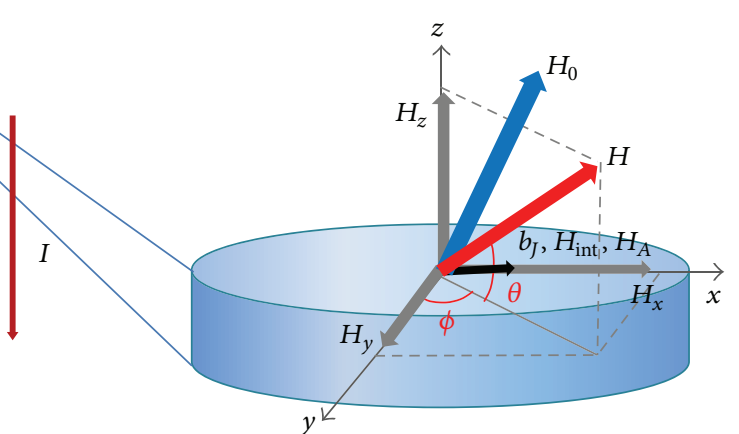

(b)

Figure 1: Simple schematic diagrams of (a) MTJ-STO device, (b) three axis components of the external and internal magnetic fields. The perpendicular torque $b_{J}$ acts like a magnetic field and is added to the direction of the fixed layer magnetization.

\section{Analytic Model of STO}

LLG equation consists of three torques which are precessional torque, damping torque, and STT as shown in the following [5]:

$$
\begin{gathered}
\frac{d \widehat{M}_{F}}{d t}=-\gamma\left[\widehat{M}_{F} \times \widehat{H}_{\mathrm{eff}}\right]+\frac{\alpha_{G}}{M_{S}}\left[\widehat{M}_{F} \times \frac{d \widehat{M}_{F}}{d t}\right]-\gamma \widehat{\tau}_{\mathrm{STT}}, \\
\widehat{\tau}_{\mathrm{STT}}=a_{J} \widehat{M}_{F} \times\left(\widehat{M}_{P} \times \widehat{M}_{F}\right)+b_{J}\left(\widehat{M}_{F} \times \widehat{M}_{p}\right) .
\end{gathered}
$$

In this equation, $\widehat{M}_{F}$ is the magnetization of the free layer, $\widehat{M}_{P}$ is the magnetization of the fixed layer, $\gamma$ is the gyromagnetic ratio, $\widehat{H}_{\text {eff }}$ is the effective magnetic field, $\alpha_{G}$ is the Gilbert damping constant, $M_{S}$ is the saturation magnetization, and $\widehat{\tau}_{\text {STT }}$ is STT.

As shown in (1b), STT consists of the in-plane torque component $a_{J}$ and the perpendicular torque component $b_{J}$. In particular, the perpendicular torque is known as the field-like torque because of its characteristics inferred from the LLG equation. The perpendicular torque acts like a magnetic field that may be added to the effective field to the direction of the fixed layer magnetization. Therefore, the effective field in the MTJ devices can be increased or decreased depending on the magnitude and the direction of the perpendicular torque. If the magnitude and the direction of the effective field are changed, the precessional motion will be changed, and the characteristics of the STO (the generation frequency, the mean oscillation power, and fullwidth-at-half-maximum (FWHM) generation linewidth) are also changed, accordingly.

The objective of our model is to precisely replicate the three characteristics of MTJ-STOs. Governing equations for those three characteristics are primarily based on Slavin's analytic model [6] which states that mean oscillation power $P_{\text {exp }}$, generation frequency $\omega_{g}$, and generation linewidth $2 \Delta \omega$ are expressed as the following equations:

$$
\begin{gathered}
P_{\exp }=R_{\mathrm{rf}} I^{2} \bar{p}, \\
\omega_{g}=\omega_{0}+N \bar{p}, \\
2 \Delta \omega=\left(1+v^{2}\right) \Gamma_{+}\left(p_{0}\right) \frac{k_{B} T}{E(\bar{p})} .
\end{gathered}
$$

In (2a), (2b), and (2c), $R_{\mathrm{rf}}$ is the magnetoresistance and defined as $R_{\mathrm{rf}}=\left(R_{L} / 2\left[R\left(\theta_{0}\right)+R_{L}\right]^{2}\right)(d R / d \theta)^{2} \cdot 4(1+\varepsilon) \cdot R_{L}$ is load resistance, and $\varepsilon$ is defined as $4 \pi M_{0} /\left(H_{0}+4 \pi M_{0}\right) \cdot \bar{p}=$ $(Q \eta /(Q+\zeta))\left[1+\exp \left(-(\zeta+Q) / Q^{2} \eta\right) / E_{\beta}\left((\zeta+Q) / Q^{2} \eta\right)\right]+((\zeta-$ $1) /(\zeta+Q))$ is the dimensionless power, $Q$ is the nonlinear damping coefficient, $\eta$ is the effective noise power, and $\zeta$ is the supercriticality parameter defined as $\left(I / I_{\mathrm{th}}\right)$. Moreover, $\beta=$ $-(1+Q) \zeta / Q^{2} \eta$ and $E_{n}(x)=\int_{1}^{\infty} e^{-x t} / t^{n} d t$ are the exponential integral functions. $\omega_{0}$ is the ferromagnetic resonance (FMR) frequency; $N$ is the nonlinear frequency shift coefficient; $v$ is the normalized dimensionless nonlinear frequency shift; $\Gamma_{+}$ $\left(p_{0}\right)$ is the positive damping rate, $T$ is the temperature, $E(\bar{p})=$ $\lambda \omega_{0} \bar{p}$ is the oscillator energy, $\lambda$ is $V_{\text {eff }} M_{0} / \gamma, V_{\text {eff }}$ is the effective volume of the oscillator, and $\gamma$ is the gyromagnetic ratio. A more detailed explanation of the parameters in (2a), (2b), and (2c) can be found in [6].

Three characteristics of an STO are determined by the bias current and external magnetic field. The field intensity, inplane angle, and out-of-plane angle of the external magnetic field affect the behavior of the STO separately. In addition, anisotropy field and coupling field (interaction field) also affect the characteristics of STO. The strength and the direction of the internal magnetic field can be calculated from the magnitude and the direction of the external magnetic field and the anisotropy field using the following equations:

$$
\begin{gathered}
H \sin \theta=H_{0} \sin \theta_{0}-4 \pi M_{0} \sin \theta, \\
H \cos \theta \cos \phi=H_{0} \cos \theta_{0} \cos \phi_{0}+H_{A} \cos \theta \cos \phi, \\
H \cos \theta \sin \phi=H_{0} \cos \theta_{0} \sin \phi_{0} .
\end{gathered}
$$

In (3a), (3b), and (3c), $H$ is the internal magnetic field magnitude. $\theta$ and $\phi$ are the internal out-of-plane angle and in-plane angle of the bias field, respectively. $H_{0}$ is the external magnetic field magnitude, and $\theta_{0}$ and $\phi_{0}$ are the external out-of-plane angle and in-plane angle of the external magnetic field, respectively. $H_{A}$ is the anisotropy field and $M_{0}$ represents saturation magnetization. The internal field is thus obtained from the external magnetic field, and the anisotropy field is used in our previous model for GMR-STO. However, as was mentioned in [7], the perpendicular torque $b_{J}$ and the coupling field $H_{\text {int }}$ should be considered together 


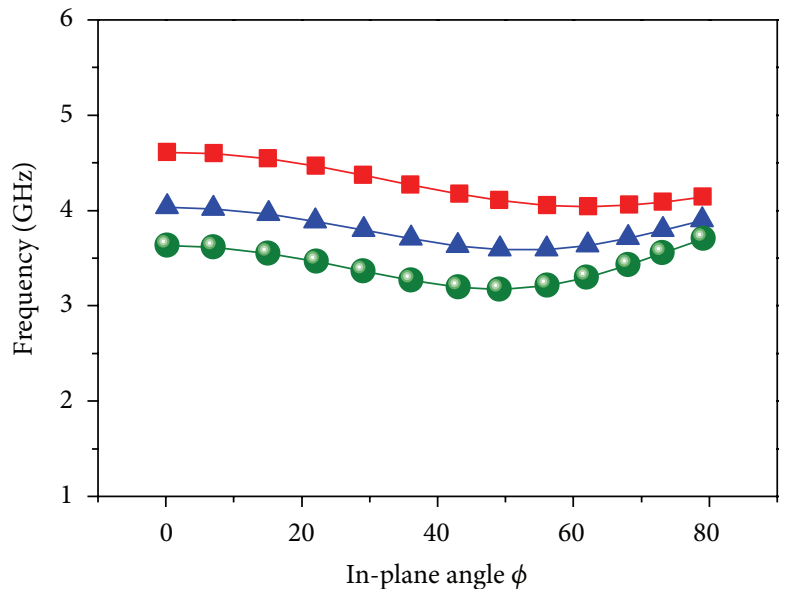

(a)

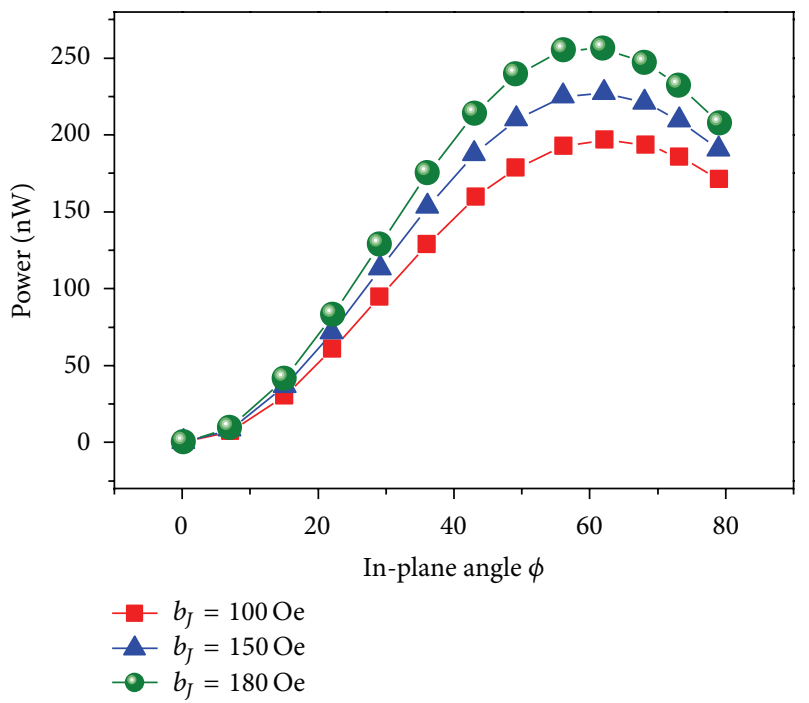

(c)

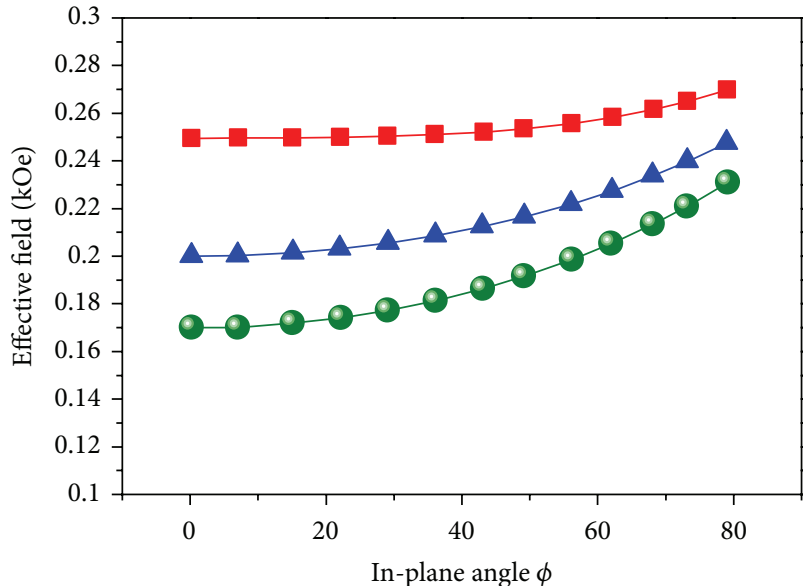

(b)

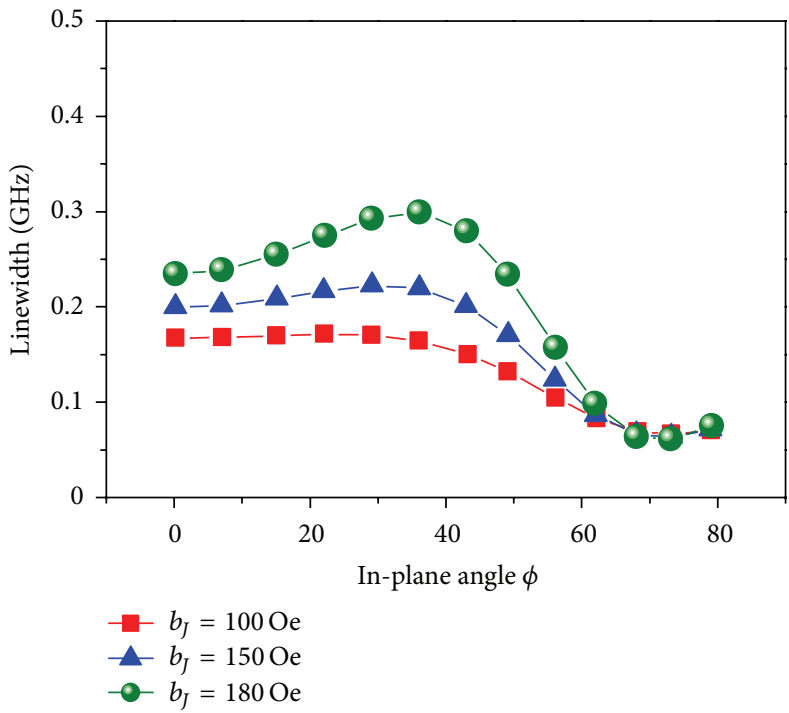

(d)

FIgURE 2: Characteristics of MTJ-STOs as functions of the perpendicular torque and the in-plane angle $\phi$ at $I=1.5 \mathrm{~mA}$. (a) Generation frequency, (b) effective field magnitude, (c) mean oscillation power and (d) generation linewidth.

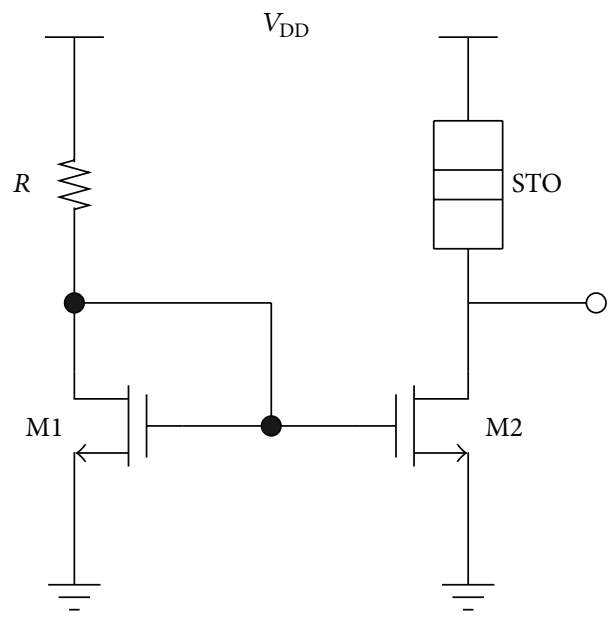

Figure 3: Current mirror circuit integrated with the MTJ-STO elements and the CMOS transistor. to accurately model the behavior of MTJ-STO devices. In the proposed model, the perpendicular torque and coupling field are included as is shown in (4a), (4b), (4c), and (4d):

$$
\begin{gathered}
H_{x_{\_} \text {new }}=H_{x}-H_{\text {int }}-b_{J}, \\
H_{\text {new }}=\sqrt{H_{x_{-} \text {new }}^{2}+H_{y}^{2}+H_{z}^{2}}, \\
\phi_{\text {new }}=\tan ^{-1}\left(\frac{H_{y}}{H_{x \_ \text {new }}}\right), \\
\theta_{\text {new }}=\tan ^{-1}\left(\frac{H_{z}}{\sqrt{H_{x_{\text {new }}}^{2}+H_{y}^{2}}}\right) .
\end{gathered}
$$

The internal magnetic field $H$ calculated in (3a), (3b), and $(3 \mathrm{c})$ is composed of three components, $H_{x}, H_{y}$, and $H_{z}$, as is shown in Figure 1(b). Both the coupling field and 


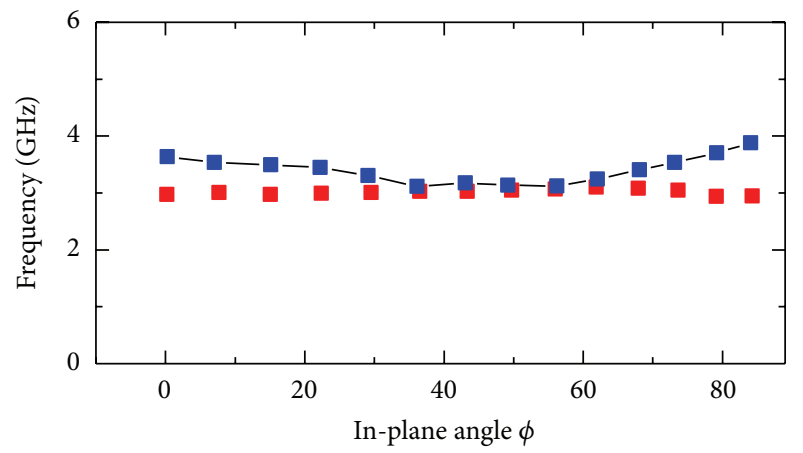

(a)

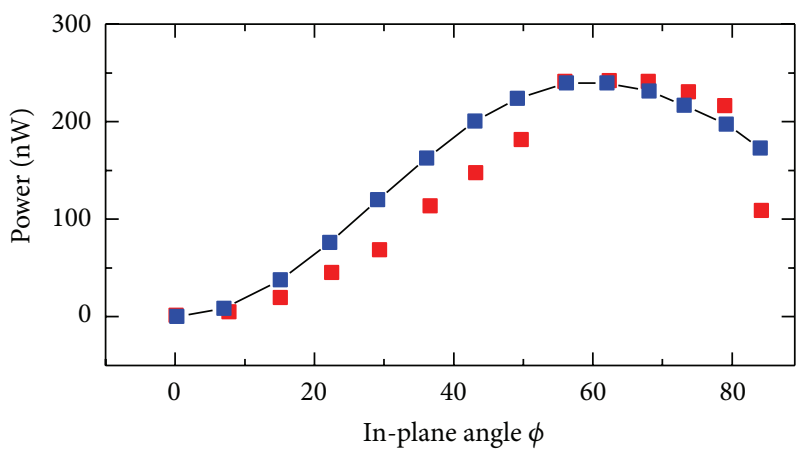

(b)

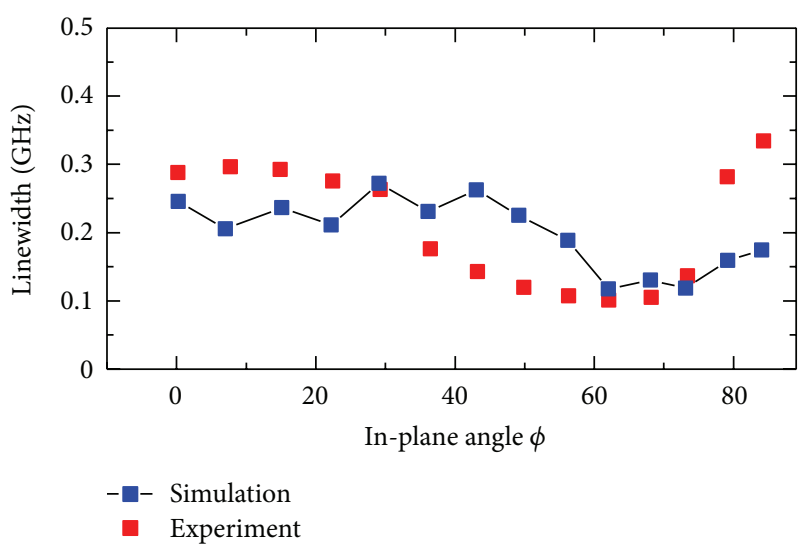

(c)

FIGURE 4: Comparison of simulation results for three characteristics of STOs with experimental data [7] as functions of the perpendicular torque and the inplane angle $\phi$. (a) Frequency, (b) mean power, and (c) linewidth.

the perpendicular torque affect the easy axis component of the internal magnetic field. Therefore, the magnitude and the direction of the new internal magnetic field $H_{\text {new }}$, $\phi_{\text {new }}$, and $\theta_{\text {new }}$ become different from the original internal magnetic field that has been calculated without considering the coupling field and the perpendicular torque. Oscillation power, generation frequency, and linewidth can now be expressed more accurately using the new internal magnetic field parameters.

The devices are assumed to be in antiparallel (AP) state as was assumed in [7]. The easy axis is set to positive $x$ axis, and the magnetization of fixed layer is towards $-x$ direction

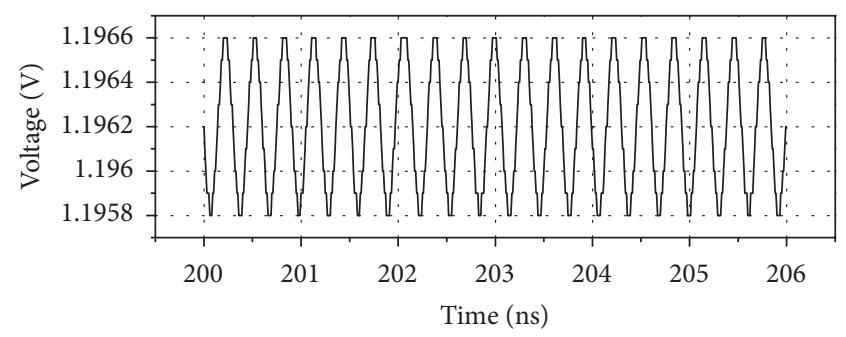

(a)

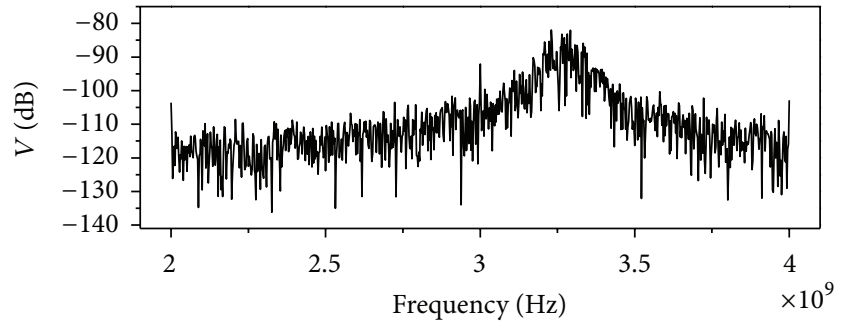

(b)

FIGURE 5: (a) The output voltage signal in the time domain, (b) the generation frequency in the frequency domain at $62^{\circ}$ of the in-plane angle.

whereas the magnetization of the free layer is set to $+x$ direction.

The perpendicular torque is known to depend on the applied voltage, but the voltage dependence of the perpendicular torque has not been clearly defined. The perpendicular torque has quadratic voltage dependence according to Sankey et al. [8] and Kubota et al. [9]. Petit et al. [10] and Li et al. [11], however, reported that the perpendicular torque has linear voltage dependence. Recently, the voltage dependence of the perpendicular torque can be generally described by the lowest order Taylor expansion $\left(b_{J}=C_{1} V+C_{2} V^{2}\right)$ that explain the main effects of asymmetric and symmetric bias dependence, simultaneously $[5,12]$. Therefore, the perpendicular torque is assumed to have linear and quadratic voltage dependence in this paper.

\section{Characteristics of STO with Perpendicular Torque}

In this section, the characteristics of an MTJ-STO versus the in-plane angle of internal magnetic field are observed while varying the magnitude of the perpendicular torque. During the simulation, the geometrical parameter and layer composition of the MTJ device are assumed to be the same as [7]. The dimension of the device is $140 \mathrm{~nm} \times 85 \mathrm{~nm}$, and the free layer thickness is $1.8 \mathrm{~nm}$. In this model, the value of the saturation magnetization and the anisotropy field are assumed to be $12 \mathrm{kOe}$ and $100 \mathrm{Oe}$, respectively. Also, as the perpendicular torque cannot be measured directly from the given experimental data, its proper value can only be found by fitting the experimental data with our MTJ-STO model by adjusting the magnitude of the perpendicular torque using $C_{1}$ and $C_{2}$. Thus, the characteristics of MTJ-STO are investigated 


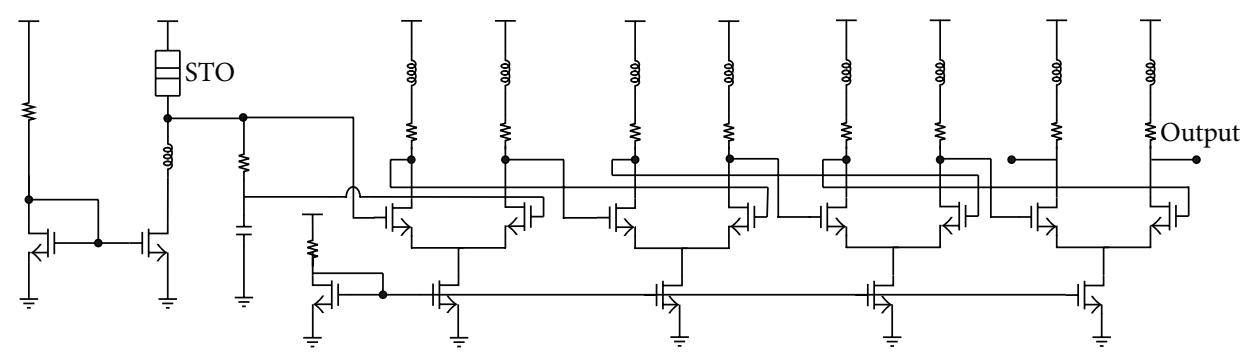

FIgURE 6: Circuit schematic of the current mirror circuit with a 4-stage common source amplifier.

by varying the magnitude of the perpendicular torque as is shown in Figure 2.

First, the generation frequency of STO as a function of the perpendicular torque is observed through the simulation. Figure 2(a) shows that the generation frequency decreases when the magnitude of the perpendicular torque increases. In particular, the frequency variation due to the perpendicular torque becomes large when the in-plane angle of the internal magnetic field is small. From the LLG equation, the perpendicular torque is added to the effective field with the direction of the magnetization of the fixed layer, so that the perpendicular torque affects the $x$ axis component of the internal effective field. Also, the $x$ axis component of the internal effective field is dominant when the in-plane angle is small. Consequently, the generation frequency decreases with increasing the perpendicular torque. In other words, the perpendicular torque tends to push the effective field to the hard axis so that the magnitude of the effective field becomes smaller at the small in-plane angle, and this phenomenon is illustrated in Figure 2(b).

Second, the mean power and the generation linewidth of the STO with perpendicular torque are observed and shown in Figures 2(c) and 2(d). Both the mean power and the generation linewidth increase with increasing the magnitude of the perpendicular torque. It is worth noting that the mean power gradually increases with increasing in-plane angle and has a maximum value at around $60^{\circ}$ while the generation linewidth decreases and has a minimum value at the point where the power has the maximum. As previously explained, the reason that the maximum power and minimum linewidth occur at a certain in-plane angle rather than the hard axis direction is the perpendicular torque which affects the effective field.

\section{Simulation Results}

Verification of our model is performed using HSPICE circuit simulator. A current mirror CMOS circuit is integrated with an MTJ-STO element as shown in Figure 3.

Proposed STO model is written in Verilog-A, which is a hardware description language for mixed-mode circuit design such that it is fully compatible with circuit-level simulators such as HSPICE. The correctness of our model was verified by comparing the simulation results with the previously reported experimental data [7]. The simulation results at $I=1.5 \mathrm{~mA}$ are shown in Figure 4 . As the magnitude of the perpendicular torque is not given with the experimental data, the different values of the perpendicular torque are tried in our simulation to find the best match. In this case, the simulation results show that the perpendicular torque of 180 Oe gives the best fit with the experimental data $\left(C_{1}=\right.$ $\left.29 \mathrm{Oe} / \mathrm{V}, C_{2}=160 \mathrm{Oe} / \mathrm{V}^{2}\right)$. The generation frequency is shown in Figure 4(a), and the simulation results show good agreement with the experimental data. In Figure 4(b), the mean power increases with increasing in-plane angle. It has the maximum value at $60^{\circ}$ both in the simulation results and the experimental data. The experimental data of the generation linewidth in Figure 4(c) shows a rapid growth at large in-plane angle. That phenomenon is due to the thermal fluctuation which is not considered in our model. The output voltage signal in the time domain and the generation frequency in the frequency domain at $62^{\circ}$ of the in-plane angle are shown in Figure 5. There are some researches that present the method for the measurement of the STT experimentally $[8,12]$; however, our model is focused on the practicality of the circuit model that expresses the characteristics of the MTJ-STO. Simply adjust the coefficient of the perpendicular torque so that the accurate representation of the MJT-STO characteristics can be possible.

Even when an MTJ-STO gives out more power than a GMR-STO, the output signal needs more amplification to be used as a high frequency microwave source to CMOS circuits. A four-stage common source wideband amplifier is designed and added to the current mirror circuit as shown in Figure 6. The bandwidth of the amplifier is about $8 \mathrm{GHz}$, and the gain is $40 \mathrm{~dB}$. Figure 7 shows the simulation results of the amplifier with our MTJ-STO model. The output spectrum of the amplifier has a same generation frequency, and the power gain is about $35 \mathrm{~dB}$.

\section{Conclusion}

In this paper, we propose a circuit-level model of MTJ-STOs. The perpendicular torque and coupling field which have been ignored in GMR-STO model are included in our new model for MTJ-STO. As the perpendicular torque magnitude increases, the force that pushes the effective magnetic field toward the hard axis becomes stronger so that the magnitude of the effective magnetic field and the generation frequency decreases accordingly. In addition, power and the linewidth increases as the perpendicular torque increases. As the perpendicular torque cannot be measured directly from the 


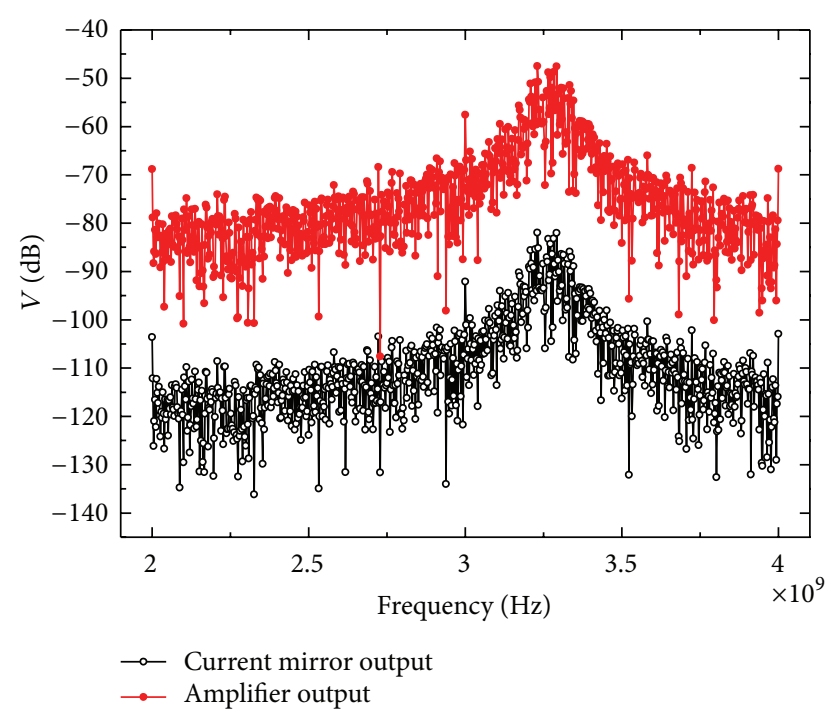

FIGURE 7: The frequency spectrum of the output signal from current mirror stage and the amplifier stage.

given experimental data, it needs to be derived from multiple simulation data with different values of the torque by bestfitting technique. In order to verify the MTJ-STO model, the current mirror CMOS circuit was composed with an MTJ-STO and simulated with HSPICE circuit simulator. The simulation results are compared with the experimental data, and they show good agreement with existing experimental data when the perpendicular torque is properly determined. Also, multistage wideband CMOS amplifier is designed and simulated with the current mirror circuit to prove the usability of our model in real circuit design environment.

As this model is based on the basic theory of microwave generation in magnetic nanostructures, it can be applied to various types of STO elements for broad application area.

\section{Acknowledgments}

This research was supported by the Basic Science Research Program through the National Research Foundation of Korea (NRF) funded by the Ministry of Education, Science, and Technology (no. 2011-0016277). And the authors would like to thank A. Slavin for the helpful discussions about the subject.

\section{References}

[1] J. Slonczewski, "Current-driven excitation of magnetic multilayers," Journal of Magnetism and Magnetic Materials, vol. 159, pp. L1-L7, 1996.

[2] L. Berger, "Emission of spin waves by a magnetic multilayer traversed by a current," Physical Review B, vol. 54, pp. 93539358, 1996.

[3] H. Lim, S. Ahn, S. Lee, and H. Shin, "Physics-based SPICE model of spin-torque oscillators," Japanese Journal of Applied Physics, vol. 51, Article ID 04DM03, 2012.

[4] S. Ahn, H. Lim, S. Lee, and H. Shin, "Analytic Model of SpinTorque Oscillators (STO) for Circuit-Level Simulation," Journal of Semiconductor Technology and Science, vol. 13, article 028, 2013.

[5] S.-C. Oh, S.-Y. Park, A. Manchon et al., "Bias-voltage dependence of perpendicular spin-transfer torque in asymmetric MgO-based magnetic tunnel junctions," Nature Physics, vol. 5, no. 12, pp. 898-902, 2009.

[6] A. Slavin and V. Tiberkevich, "Nonlinear auto-oscillator theory of microwave generation by spin-polarized current," IEEE Transactions on Magnetics, vol. 45, no. 4, pp. 1875-1918, 2009.

[7] Z. M. Zeng, P. Upadhyaya, P. Khalili Amiri et al., "Enhancement of microwave emission in magnetic tunnel junction oscillators through in-plane field orientation," Applied Physics Letters, vol. 99, no. 3, Article ID 032503, 2011.

[8] J. C. Sankey, Y.-T. Cui, J. Z. Sun, J. C. Slonczewski, R. A. Buhrman, and D. C. Ralph, "Measurement of the spin-transfertorque vector in magnetic tunnel junctions," Nature Physics, vol. 4, no. 1, pp. 67-71, 2008.

[9] H. Kubota, A. Fukushima, K. Yakushiji et al., "Quantitative measurement of voltage dependence of spin-transfer torque in MgO-based magnetic tunnel junctions," Nature Physics, vol. 4, no. 1, pp. 37-41, 2008.

[10] S. Petit, C. Baraduc, C. Thirion et al., "Spin-torque influence on the high-frequency magnetization fluctuations in magnetic tunnel junctions," Physical Review Letters, vol. 98, no. 7, Article ID 077203, 2007.

[11] Z. Li, S. Zhang, Z. Diao et al., "Perpendicular spin torques in magnetic tunnel junctions," Physical Review Letters, vol. 100, no. 24, Article ID 246602, 2008.

[12] C. Wang, Y.-T. Cui, J. A. Katine, R. A. Buhrman, and D. C. Ralph, "Time-resolved measurement of spin-transfer-driven ferromagnetic resonance and spin torque in magnetic tunnel junctions," Nature Physics, vol. 7, no. 6, pp. 496-501, 2011. 

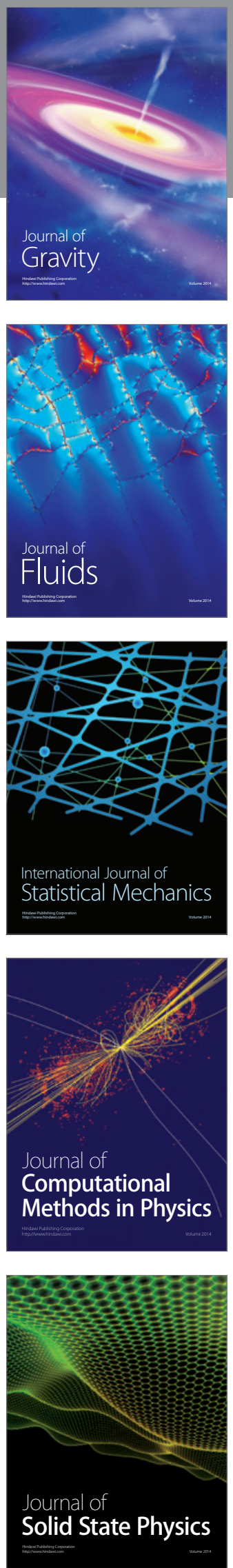

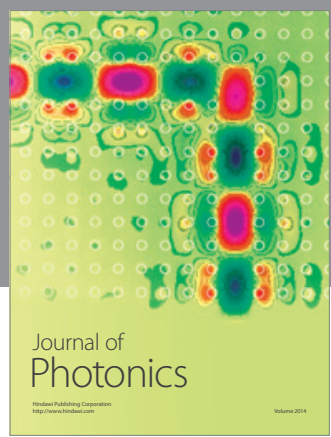

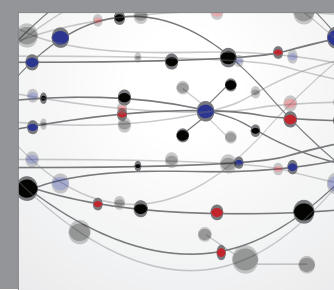

The Scientific World Journal

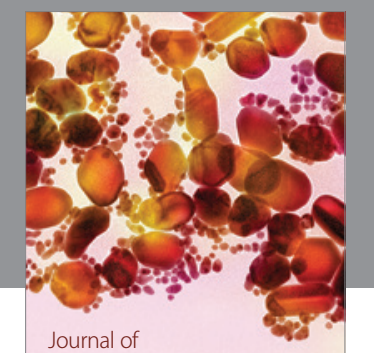

Soft Matter
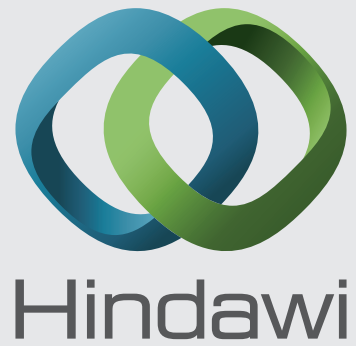

Submit your manuscripts at

http://www.hindawi.com
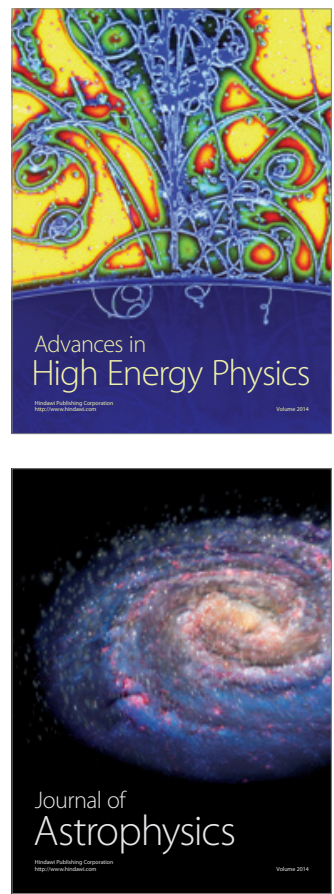
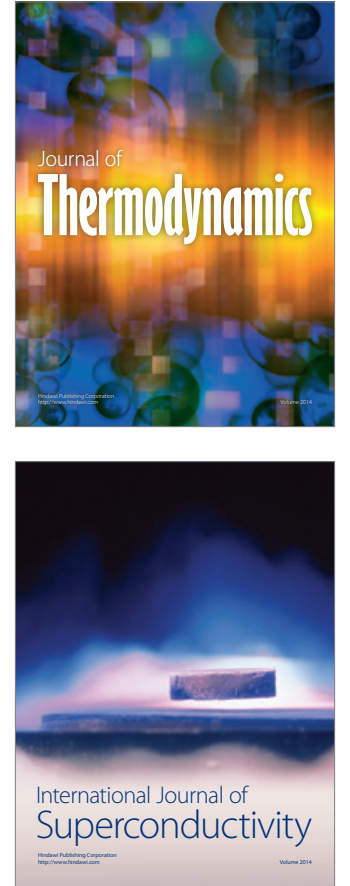
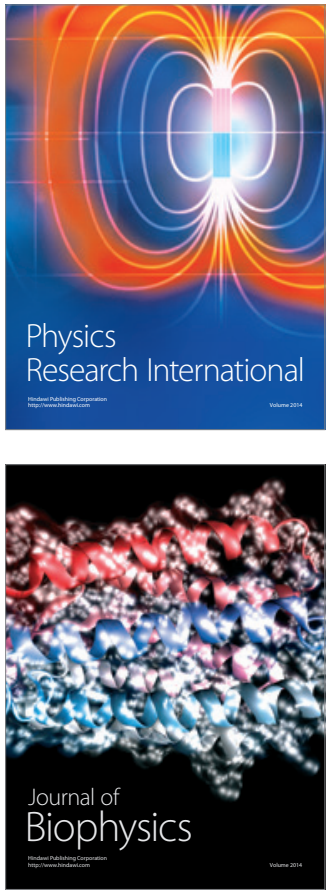
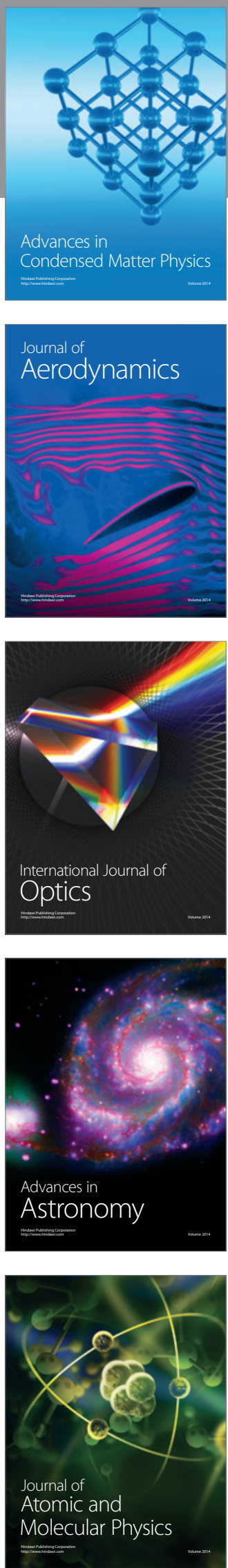